\title{
Teste de condutividade elétrica para avaliação do vigor em sementes de quinoa
}

\author{
Electrical conductivity test to assess vigor in quinoa seeds \\ Prueba de conductividad eléctrica para evaluar el vigor en semillas de quinua
}

Recebido: 05/04/2021 | Revisado: 12/04/2021 | Aceito: 15/04/2021 | Publicado: 01/05/2021

\author{
Janete Denardi Munareto \\ ORCID: https://orcid.org/0000-0002-3724-168X \\ Universidade Federal de Santa Maria, Brasil \\ E-mail: jdmunareto@gmail.com \\ Sandro Luis Petter Medeiros \\ ORCID: https://orcid.org/0000-0003-2603-480X \\ Universidade Federal de Santa Maria, Brasil \\ E-mail: slpmedeiros@yahoo.com.br \\ Ubirajara Russi Nunes \\ ORCID: https://orcid.org/0000-0002-7124-9204 \\ Universidade Federal de Santa Maria, Brasil \\ E-mail: russinunes@yahoo.com.br \\ Edmar Soares de Vasconcelos \\ ORCID: https://orcid.org/0000-0002-0481-9879 \\ Universidade Estadual do Oeste do Paraná, Brasil \\ E-mail: edmar.vasconcelos@unioeste.br \\ Janine Farias Menegaes \\ ORCID: https://orcid.org/0000-0001-6053-4221 \\ Universidade Federal de Santa Maria, Brasil \\ E-mail: janine_rs@hotmail.com
}

\begin{abstract}
Resumo
A quinoa destaca-se por apresentar alto potencial nutricional e capacidade de adaptação a diferentes ambientes. Todavia, a qualidade das sementes é um dos principais fatores a ser considerado na implantação da cultura, desta forma tornam-se importante complementar as informações fornecidas pelo teste de germinação através de testes de vigor. Deste modo, objetivou-se adequar a metodologia do teste de condutividade elétrica para a avaliação da qualidade fisiológica de sementes quinoa. A caracterização inicial dos lotes de sementes foi realizada através do teor de água inicial, massa de mil sementes, testes de germinação e de emergência de plântulas em substrato comercial. Para o teste de condutividade elétrica foram utilizados quatro lotes de sementes (genótipo Q13-31) cultivadas nas safras de 2016/2017 e 2017/2018, empregando-se 25, 50 e 75 sementes, a 20 C, em volumes de água deionizada (25 e $50 \mathrm{~mL}$ ), por períodos de embebição $(1,2,3,4$ e $5 \mathrm{~h})$. O delineamento foi o inteiramente casualizado, as médias analisadas pelo teste Scott-Knott. Realizou-se análise de correlação simples entre a condutividade elétrica e os testes de germinação e de emergência de plântulas. O teste de condutividade elétrica conduzido com 50 sementes embebidas em $50 \mathrm{~mL}$ de água deionizada, durante $2 \mathrm{~h}$, é eficiente para a avaliação do potencial fisiológico dos lotes de sementes de quinoa, além de apresentar correlações significativas com o teste de emergência.
\end{abstract}

Palavras-chave: Chenopodium quinoa Willd; Germinação; Períodos de embebição.

\begin{abstract}
Quinoa stands out for its high nutritional potential and its ability to adapt to different environments. However, seed quality is one of the main factors to be considered when implanting the crop, so it becomes important to complement the information provided by the germination test through vigor tests. Thus, the objective was to adapt the methodology of the electrical conductivity test to assess the physiological quality of quinoa seeds. The initial characterization of the seed lots was carried out through the initial water content, mass of a thousand seeds, germination and seedling emergence tests in commercial substrate. For the electrical conductivity test, four seed lots (genotype Q13-31) were grown in the 2016/2017 and 2017/2018 seasons, using 25, 50 and 75 seeds, at $20^{\circ} \mathrm{C}$, in volumes of deionized water $(25$ and $50 \mathrm{~mL}$ ), for periods of imbibition (1, 2, 3, 4 and $5 \mathrm{~h}$ ). The design was completely randomized, the means analyzed by the Scott-Knott test. A simple correlation analysis was carried out between electrical conductivity and germination and seedling emergence tests. The electrical conductivity test conducted with 50 seeds soaked in $50 \mathrm{~mL}$ of deionized water, for $2 \mathrm{~h}$, is efficient for the evaluation of the physiological potential of the quinoa seed lots, in addition to presenting significant correlations with the emergency test.
\end{abstract}

Keywords: Chenopodium quinoa Willd; Germination; Imbibition periods. 


\begin{abstract}
Resumen
La quinua destaca por su alto potencial nutricional y su capacidad para adaptarse a diferentes ambientes. Sin embargo, la calidad de la semilla es uno de los principales factores a tener en cuenta a la hora de implantar el cultivo, por lo que se vuelve importante complementar la información que brinda la prueba de germinación mediante pruebas de vigor. Así, el objetivo fue adaptar la metodología de prueba de conductividad eléctrica para evaluar la calidad fisiológica de las semillas de quinua. La caracterización inicial de los lotes de semillas se realizó mediante el contenido inicial de agua, masa de mil semillas, pruebas de germinación y emergencia de plántulas en sustrato comercial. Para la prueba de conductividad eléctrica se cultivaron cuatro lotes de semillas (genotipo Q13-31) en las temporadas 2016/2017 y 2017/2018, utilizando 25,50 y 75 semillas, a $20^{\circ} \mathrm{C}$, en volúmenes de agua desionizada ( 25 y $50 \mathrm{~mL}$ ), para periodos de imbibición (1, 2, 3, 4 y 5 h). El diseño fue completamente al azar, las medias analizadas por la prueba de ScottKnott. Se realizó un análisis de correlación simple entre la conductividad eléctrica y las pruebas de germinación y emergencia de plántulas. La prueba de conductividad eléctrica realizada con 50 semillas remojadas en $50 \mathrm{~mL}$ de agua desionizada, durante $2 \mathrm{~h}$, es eficiente para la evaluación del potencial fisiológico de los lotes de semillas de quinua, además de mostrar correlaciones significativas con la prueba de emergencia.
\end{abstract}

Palabras clave: Chenopodium quinoa Willd; Germinación; Periodos de imbibición.

\title{
1. Introdução
}

A quinoa (Chenopodium quinoa Willd.) é uma cultura anual, nativa do Altiplano Andino, entre a Bolívia e Peru, sendo esses os principais produtores e exportadores de grãos desta espécie. Caracteriza-se como uma planta versátil do ponto de vista alimentar e agronômico. Seus grãos são altamente nutritivos, contém baixos níveis de colesterol, alto teor de fibras, ausência de glúten (Vega-Gálvez et al., 2010). Além disso, contêm todos os aminoácidos essenciais, compostos fenólicos e peptídeos bioativos que apresentam efeitos benéficos na saúde cardiovascular e gastrointestina (Vilcacundo et al., 2017). A ampla variabilidade genética permite que essa espécie seja cultivada em condições ambientes variados em relação a solo, temperatura, seca, altitude, entre outros (Murphy et al. 2016).

$\mathrm{Na}$ última década, a cultura se expandiu em todos os continentes, dando origem aos estudos que investigam o potencial do cultivo em novos ambientes fora da região andina. Diversos países da Europa, África, Ásia, América do Norte e do Sul, estão buscando obter genótipos adaptados as suas condições climática (Bazile et al., 2016). No Brasil, esses estudos iniciaram na década de 1990, dando origem a duas cultivares BRS Piabiru e Syetetuba, com potencial produtivo de até $3 \mathrm{t} \mathrm{ha}^{-1}$ (Spehar et al., 2011). Todavia, a produção brasileira é ainda incipiente, faltando informações básicas, como a qualidade de sementes.

O uso de sementes de qualidade é um fator determinante na implantação de uma cultura, uma vez que esta pode ser afetada por diversos fatores ambientais durante todas as etapas de cultivo até o armazenamento de sementes. Entre os atributos da semente, o potencial fisiológico é fundamental no controle de qualidade realizado por testes de rotina laboratorial e campo, e o aprimoramento desses deve ser constante (Mattioni et al., 2015), principalmente em cultura que não possuem regras de análise definidas como a quinoa. Teste de vigor tem por finalidade estimar o potencial germinativo da semente, em condições adversas de campo. Entre eles, o teste de condutividade elétrica é considerado eficiente na determinação do potencial fisiológico de sementes. Por avaliar indiretamente os danos nas membranas celulares da semente resultantes do processo de deterioração, esse teste quantifica os íons lixiviados (açúcares, aminoácidos, ácidos graxos, proteínas, enzimas, entre outros) durante o processo de embebição (Marcos Filho, 2015). Lotes de sementes que apresentam elevados índices de eletrólitos lixiviados para o meio externo, apresentam baixo potencial germinativo e de emergência no campo (Marcos Filho, 2015; Powell \& Mavi, 2016).

A execução do teste de condutividade elétrica apesar de ser simples, rápido e confiável, é padronizado pela Internacional Seed Testing Association (ISTA) para espécies de sementeiras como ervilha (Pisum sativum L.), soja (Glycine $\max$ L.), feijão (Phaseolus vulgaris L.) e grão-de-bico (Cicer arietinum L.).

Neste contexto, pelo grande potencial agroeconômico da cultura de quinoa, o objetivo deste trabalho foi adequar a metodologia do teste de condutividade elétrica para a avaliação da qualidade fisiológica de sementes quinoa. 


\section{Metodologia}

O trabalho foi realizado, na área experimental e as avaliações com sementes foram realizadas no Laboratório Didático e de Pesquisa em Sementes do Departamento de Fitotecnia da Universidade Federal de Santa Maria, Santa Maria, RS (29 ${ }^{\circ} 3^{\prime}$ S; 534' W e altitude de 95m). O clima na região é subtropical úmido (Cfa), segundo a classificação de Köppen-Geiger, com precipitação média anual acumulada de $1.769 \mathrm{~mm}$, temperatura média anual próxima de $19,2^{\circ} \mathrm{C}$ e umidade do ar em torno de $78,4 \%$.

Os lotes de sementes de quinoa do genótipo Q13-31, são oriundos de experimentos com quatro épocas de semeaduras realizadas nos períodos de 01/12/2016 (lote 1), 01/05/2017 (lote 2), 07/10/2017 (lote 3) e em 09/11/2017 (lote 4). Depois de cada colheita, as sementes foram armazenadas em câmara fria $\left(15^{\circ} \mathrm{C}\right.$ e $\left.40 \% \mathrm{UR}\right)$ em sacos de papel Kraft (tipo pardo de 1,0 $\mathrm{kg}$ ), com grau de umidade médio de $12,0 \%$ até a execução do experimento.

Para comparar e diferenciar o potencial fisiológico dos lotes, antes do teste de condutividade elétrica, as sementes foram submetidas aos seguintes testes:

a) Teor de água das sementes (TAI), determinado pelo método de estufa a $105 \pm 3^{\circ} \mathrm{C}$, por $24 \mathrm{~h}$, utilizando-se duas repetições de 2,0 g de sementes para cada lote (Brasil, 2009).

b) Massa de mil sementes (MMS): foram utilizadas oito subamostras de 100 sementes de cada lote, adaptado de Brasil (2009).

c) Germinação $(G)$ : para cada lote, foram utilizadas quatro repetições de 50 sementes distribuídas sobre duas folhas de papel de germinação (tipo germitest) em caixas plásticas tipo gerbox, umedecidas com água destilada (2,5 vezes massa do substrato seco). As sementes foram mantidas em câmara de germinação tipo Box Organism Development (B.O.D.) a $20^{\circ} \mathrm{C}$, com luz. Aos quatro dias após a semeadura (DAS) foi avaliada a primeira contagem, e, no sexto DAS a contagem final de plântulas germinadas, conforme Borges (2017) e os resultados expressos em porcentagens de plântulas normais seguindo os critérios estabelecidos nas Regras para Análise de Sementes (BRASIL, 2009).

d) Emergência de plântulas em casa de vegetação (EP): foram semeadas quatro repetições de 50 sementes por lote, em bandejas de propileno, com substrato comercial Plantmax ${ }^{\circledR}$, e as sementes distribuídas em sulcos a 1,0 cm de profundidade em fileiras espaçada a 5,0 cm. A contagem de plântulas emergidas foi feita no décimo quarto dia após a semeadura, quando não foi mais observado emergência de novas plântulas.

Após a caracterização da qualidade inicial dos lotes de sementes, foram testadas metodologias para condução do teste de condutividade elétrica (CE) pelo método massal, organizado em esquema fatorial 4x5 (lotes de sementes e períodos de embebição). Os lotes de sementes são os supracitados e, as combinações foram compostas por períodos de embebição (1, 2, 3, 4 e 5 h). Para a execução do teste de CE empregou-se a combinação dos números de sementes (25, 50 e 75 sementes) com os volumes de água (25 e $50 \mathrm{~mL}$ ) adaptado de Araujo et al. (2011).

Inicialmente realizou-se a contagem manual das sementes e a sua respectiva pesagem em balança digital $(0,001 \mathrm{~g})$. $\mathrm{Na}$ sequência do experimento as sementes foram dispostas em copos plásticos $(200 \mathrm{~mL})$, contendo água deionizada nos volumes pré-determinados e mantidos em câmara B.O.D a $20^{\circ} \mathrm{C}$ durante os períodos de embebição. Após cada período de embebição, a condutividade elétrica da solução, foi mensurada em condutivímetro DIGIMED DM-31, e os valores médios, para cada lote, expressos em $\mu \mathrm{S} \mathrm{cm}^{-1} \mathrm{~g}^{-1}$.

Os dados em porcentagem foram transformados em arco-seno $\sqrt{\%} / 100$ e analisados pelo Software Sisvar ${ }^{\circledR}$ (Ferreira, 2014), e as médias foram comparadas pelo teste Skott-Knott ( $\mathrm{p}<0,05)$. Os dados referentes ao teor de água inicial das sementes não foram submetidos análise estatística. A correlação de Pearson $(p<0,01)$ e $(p<0,05)$ entre o teste de condutividade 
elétrica e os testes de primeira contagem, germinação e emergência de plântulas, foi realizado através do programa R (R CORE TEAM, 2018).

\section{Resultados e Discussão}

Os teores de água inicial (TAI) dos quatro lotes de sementes testados foram semelhantes e variaram somente em 0,5\% entre si (Tabela 1). Esta variação no TAI foi inferior a $2 \%$, considerado por Marcos Filho (2015), como um fator importante na padronização de um método, bem como na o obtenção de resultados confiáveis e consistentes.

Em relação a massa de mil sementes MMS (Tabela 1), o lote 1 destacou-se como melhor em comparação aos demais, classificando o lote 2 como o de pior qualidade, enquanto que os lotes 3 e 4 foram agrupados num mesmo nível intermediário, não diferindo entre si. O lote 1 destacou-se com 23\% a mais de massa que o lote 2. Segundo Carvalho e Nakagawa (2012), sementes com maior MMS, além de armazenarem maior quantidade de reservas (carboidratos, lipídeos e proteínas) possuem capacidade de mobilizar estas substâncias e gerar plântulas vigorosas com elevados índices de germinação nos testes de primeira contagem (PC), germinação (G) e emergência de plântulas (EP) (Tabela 1).

Tabela 1 - Caracterização dos lotes de sementes de quinoa (C. quinoa). Teor de água inicial (TAI), massa de mil sementes (MMS), primeira contagem de germinação (PC), germinação $(\mathrm{G})$ e emergência de plântulas (EP).

\begin{tabular}{cccccc}
\hline Lotes & TAI (\%) & MMS (g) & PC (\%) & G (\%) & EP (\%) \\
\hline 1 & 12,0 & $3,2 \mathrm{a}^{*}$ & $96 \mathrm{a}$ & $96 \mathrm{a}$ & $74 \mathrm{~b}$ \\
2 & 12,2 & $2,6 \mathrm{c}$ & $72 \mathrm{c}$ & $96 \mathrm{c}$ & $90 \mathrm{a}$ \\
3 & 12,3 & $2,8 \mathrm{~b}$ & $96 \mathrm{a}$ & $85 \mathrm{~b}$ & $82 \mathrm{~b}$ \\
\hline Médias & 12,5 & $2,8 \mathrm{~b}$ & 87,2 & 89 & 85 \\
\hline CV(\%) & 12 & 2,9 & 6,5 & 7,1 & 8,5 \\
\hline
\end{tabular}

*Médias seguidas pela mesma letra, pertencem ao mesmo grupo pelo Teste Sckott-Knott $(\mathrm{p}<0,05)$. Fonte: Autores.

Verificou-se que a germinação dos lotes foi semelhante, exceto para o lote 2 (Tabela 1) confirmando a inabilidade do teste em não detectar diferenças na qualidade de lotes com germinação semelhante, ou seja, Lotes 1, 3 e 4. De acordo com Delouche e Baskin (1973), a dificuldade do teste de germinação em identificar diferenças no comportamento dos lotes com germinação semelhante ocorre, pois, as primeiras manifestações, são relacionadas à deterioração, geralmente ocorrem, antes que sejam observados declínios na capacidade germinativa dos lotes. Por isso, testes de vigor são fundamentais no monitoramento da qualidade das sementes a partir da maturidade fisiológica (Marcos Filho, 2015).

Os testes de PC e EP (Tabela 1) constituem-se em parâmetros indicadores da eficiência dos testes para avaliar a qualidade fisiológica, identificando as diferenças de vigor entre os lotes, que o teste de germinação não identificou evidenciando a importância de efetuar vários testes. Os lotes 1 e 3 destacaram-se como mais vigorosos que os demais lotes estudados, com percentuais de germinação acima de 90\%, enquanto que o lote 4 apresentou qualidade intermediária (83\%) e o lote 2 com o pior desempenho, com média de 70\% de germinação.

De acordo com ISTA (2014) e Marcos Filho (2015), esses indicam que a sensibilidade dos testes de PC e EP são fatores importantes, pois os testes de PC identificam a capacidade do lote se estabelecer de forma rápida, e os testes de EP confirmam o potencial do lote de se estabelecer no campo em condições ambientais não ideais para a espécie. A sensibilidade dos testes PC e EP foram relatadas por Torres et al. (2012; 2015) em sementes de coentro (Coriandrum sativum L.), Lodo et al. (2013) em espinafre (Spinacia oleracea L.) e Sponchiado et al. (2014) em aveia preta (Avena strigosa Schreb). 
Observou-se que, quando o teste de condutividade elétrica (CE) (Tabela 2) foi conduzido com 25 ou 75 sementes independente do volume de água utilizado, não apresentou interação significativa entre lotes de sementes e períodos de embebição. Todavia, ao analisar as médias de cada fator de forma isolada, o teste destacou o lote 1 como sendo o de melhor qualidade, os lotes 3 e 4 como intermediárias e o lote 2 com a pior qualidade fisiológica.

Quando o teste de CE (Tabela 2) foi conduzido com 50 sementes e $25 \mathrm{~mL}$ de água deionizada, diferenciou a qualidade dos lotes em todos os períodos de embebição. Assim, como observado na caracterização inicial, o teste de CE confirmou os lotes 1 e 2 como de qualidade superior e inferior respectivamente. No entanto, a discriminação dos lotes 3 e 4 não foi coerente com resultados dos testes de PC e EP (Tabelas 1 e 2).

A classificação dos lotes de forma semelhante aos obtidos nos testes de PC e EP (Tabelas 1 e 2) ficou evidente quando o teste de CE foi realizado com $50 \mathrm{sem} / 50 \mathrm{~mL}$ com $2 \mathrm{~h}$ de embebição. O período de $2 \mathrm{~h}$ foi mais efetivo, pois no início da embebição, liberação de eletrólitos é intensa, tanto em sementes vigorosas como nas deterioradas, dificultando identificar, de forma segura, possíveis diferenças de vigor entre os lotes (Rosa et al., 2000). Com o decorrer desse processo, sementes vigorosas tendem a reparar as membranas de forma mais rápida, que as de baixo vigor, reduzindo o vazamento de eletrólitos, e quantidade de solutos liberados tende a se estabilizar, possibilitando a separação dos lotes em diferentes níveis de vigor (Menezes et al., 2007). Este resultado mostra-se coerente com os observados por Torres et al. (2015) avaliando sementes de coentro, utilizando 50 sementes $/ 50 \mathrm{~mL}$ de água, em $2 \mathrm{~h}$, conseguiram ordenar os lotes diferentes níveis de qualidade fisiológica.

Em relação ao período de embebição, verificou-se que em todos os tratamentos aumentaram a quantidade de eletrólitos liberados pelas sementes com o decorrer do período de embebição (Tabela 2) conforme relatado por Haesbaert et al. (2017), quanto mais tempo as sementes permanecem imersas em água, mais fluídas as membranas ficam e consequentemente, liberam maiores quantidade de solutos para meio externo, resultando em aumentos nos valores de CE.

$\mathrm{O}$ valor de condutividade, no menor volume de água utilizado $(25 \mathrm{~mL})$, independentemente do tempo e do número de sementes utilizadas em todos os tratamentos, foi superior no maior volume $(50 \mathrm{~mL})$, devido a maior concentração dos lixiviados no menor volume de embebição. Resultados semelhantes foram obtidos em sementes de rúcula (Eruca sativa L.) (Torres \& Pereira, 2010) e girassol (Helianthus annuиs L.) (Haesbaert et al. (2017). 
Research, Society and Development, v. 10, n. 5, e12510514682, 2021

(CC BY 4.0) | ISSN 2525-3409 | DOI: http://dx.doi.org/10.33448/rsd-v10i5.14682

Tabela 2 - Condutividade elétrica em função do número de sementes e volume de água, períodos de embebição de sementes de quinoa (C. quinoa).

\begin{tabular}{|c|c|c|c|c|c|c|}
\hline \multirow{2}{*}{ Lotes } & \multicolumn{6}{|c|}{ Períodos de embebição (h) } \\
\hline & 1 & 2 & 3 & 4 & 5 & Média \\
\hline \multicolumn{7}{|c|}{25 sementes / $25 \mathrm{~mL}\left(\mu \mathrm{S} \mathrm{cm}^{-1} \mathrm{~g}^{-1}\right)$} \\
\hline 1 & $328, .5$ & 409,8 & 448,6 & 477 & 539,8 & $440,8 \mathrm{~A}$ \\
\hline 2 & 549 & 641,7 & 683,9 & 708,1 & 718,6 & $660,3 \mathrm{D}$ \\
\hline 3 & 465,2 & 548,5 & 603,6 & 676,1 & 692,2 & $597,1 \mathrm{C}$ \\
\hline 4 & 482,5 & 538,7 & 570 & 613,2 & 627,2 & $566,3 \mathrm{~B}$ \\
\hline Média & $456,3 \mathrm{a}$ & $534,7 \mathrm{~b}$ & $576,5 \mathrm{c}$ & $618,6 \mathrm{~d}$ & $644,4 \mathrm{~d}$ & \\
\hline $\mathrm{CV}(\%)$ & 7.0 & & & & & \\
\hline \multicolumn{7}{|c|}{25 sementes / $50 \mathrm{~mL}\left(\mu \mathrm{S} \mathrm{cm}^{-1} \mathrm{~g}^{-1}\right)$} \\
\hline 1 & 198,3 & 241,4 & 270,7 & 285,2 & 294,8 & $258,1 \mathrm{~A}$ \\
\hline 2 & 305,6 & 353,2 & 375,1 & 387,4 & 402,7 & $364,8 \mathrm{C}$ \\
\hline 3 & 242,7 & 296,2 & 342,6 & 345,4 & 367,6 & $318,9 \mathrm{~B}$ \\
\hline 4 & 259,1 & 301,3 & 324,7 & 338,8 & 346,7 & $314,1 \mathrm{~B}$ \\
\hline Média & $251,4 \mathrm{a}$ & $298,0 \mathrm{~b}$ & $328,3 \mathrm{c}$ & $339,2 \mathrm{c}$ & $353,0 \mathrm{~d}$ & \\
\hline $\mathrm{CV}(\%)$ & 6,1 & & & & & \\
\hline \multicolumn{7}{|c|}{50 sementes / $25 \mathrm{~mL}\left(\mu \mathrm{S} \mathrm{cm}^{-1} \mathrm{~g}^{-1}\right)$} \\
\hline 1 & $328,3 \mathrm{Aa}$ & $393,6 \mathrm{Ba}$ & $431,8 \mathrm{Ca}$ & $451,7 \mathrm{Ca}$ & $471,6 \mathrm{Ca}$ & 415,4 \\
\hline 2 & $525,2 \mathrm{Ac}$ & $619,9 \mathrm{Bd}$ & $665,5 \mathrm{Cd}$ & $696,3 \mathrm{Dd}$ & $719,3 \mathrm{Dd}$ & 645,2 \\
\hline 3 & $378,9 \mathrm{Ab}$ & $482,6 \mathrm{Bb}$ & $524,8 \mathrm{Cb}$ & $559,1 \mathrm{Db}$ & $576,4 \mathrm{Db}$ & 504,4 \\
\hline 4 & $397,7 \mathrm{Ab}$ & $571,5 \mathrm{Bc}$ & $624,6 \mathrm{Cc}$ & $641,5 \mathrm{Cc}$ & $658,8 \mathrm{Cc}$ & 578,8 \\
\hline Média & 407,5 & 516,9 & 561,7 & 587,2 & 606,5 & \\
\hline $\mathrm{CV}(\%)$ & 4,7 & & & & & \\
\hline \multicolumn{7}{|c|}{50 sementes $/ 50 \mathrm{~mL}\left(\mu \mathrm{S} \mathrm{cm}^{-1} \mathrm{~g}_{-}{ }^{1}\right)$} \\
\hline 1 & $186,7 \mathrm{Aa}$ & $223,3 \mathrm{Ba}$ & $243,0 \mathrm{Ca}$ & $256,1 \mathrm{Da}$ & $266,5 \mathrm{Da}$ & 235,1 \\
\hline 2 & $231,4 \mathrm{Ab}$ & $297,3 \mathrm{Bc}$ & $335,5 \mathrm{Cc}$ & $354,5 \mathrm{Dc}$ & $370,2 \mathrm{Dd}$ & 317,8 \\
\hline 3 & $218,9 \mathrm{Ab}$ & $225,3 \mathrm{Ba}$ & $269,0 \mathrm{Cb}$ & $307,4 \mathrm{Db}$ & $331,5 \mathrm{Ec}$ & 270,4 \\
\hline 4 & 196,4 Aa & $252,9 \mathrm{Bb}$ & $281,5 \mathrm{Cb}$ & $296,2 \mathrm{Cb}$ & $310,8 \mathrm{Cb}$ & 267,6 \\
\hline Média & 208,4 & 249,7 & 282,3 & 303,6 & 319,8 & \\
\hline $\mathrm{CV}(\%)$ & 5,7 & & & & & \\
\hline \multicolumn{7}{|c|}{75 sementes $/ 25 \mathrm{~mL}\left(\mu \mathrm{S} \mathrm{cm}^{-1} \mathrm{~g}^{-1}\right)$} \\
\hline 1 & 271,0 & 363,8 & 406,8 & 431,8 & 447,5 & $384,2 \mathrm{~A}$ \\
\hline 2 & 407,5 & 536,1 & 585,6 & 615,6 & 629,7 & $554,9 \mathrm{D}$ \\
\hline 3 & 360,6 & 476,7 & 539,7 & 559,8 & 582,6 & $503,9 \mathrm{C}$ \\
\hline 4 & 391,8 & 460,6 & 496,4 & 535 & 549 & $486,6 \mathrm{~B}$ \\
\hline Média & 357,7 a & $459,3 \mathrm{~b}$ & $507,1 \mathrm{c}$ & $535,5 \mathrm{~d}$ & $552,2 \mathrm{~d}$ & \\
\hline $\mathrm{CV}(\%)$ & 5,5 & & & & & \\
\hline \multicolumn{7}{|c|}{75 sementes $/ 50 \mathrm{~mL}\left(\mu \mathrm{S} \mathrm{cm}^{-1} \mathrm{~g}^{-1}\right)$} \\
\hline 1 & 151,9 & 199,1 & 223,4 & 236,2 & 245,4 & $211,2 \mathrm{~A}$ \\
\hline 2 & 209,6 & 27,7 & 296,2 & 312,5 & 323,5 & $282,5 \mathrm{C}$ \\
\hline 3 & 155,1 & 218,5 & 255,1 & 273,5 & 283,8 & $237,2 \mathrm{~B}$ \\
\hline 4 & 158,9 & 222,8 & 259,8 & 274,5 & 284,3 & $240,1 \mathrm{~B}$ \\
\hline Média & $168,9 \mathrm{a}$ & $227,8 \mathrm{~b}$ & $258,6 \mathrm{c}$ & $274,2 \mathrm{~d}$ & $284,3 \mathrm{e}$ & \\
\hline
\end{tabular}

* Médias seguidas pela mesma letra maiúscula na linha e minúscula na coluna, não diferem entre si pelo teste de $\operatorname{Scott}-\mathrm{Knott}(\mathrm{p}<0,05)$. Fonte: Autores. 
A correlação entre os testes de CE e os testes de PC, G e EP, apresentou resultados negativo, indicando que à medida que aumenta a condutividade diminuiu o vigor, ou seja, quanto maior os níveis de lixiviação, menor será a qualidade fisiológica das sementes (Tabela 3). Em todas as combinações do número de sementes e volume de água, o coeficiente foi significativo para o teste de EP. As maiores correlações foram encontradas entre teste de CE e EP em $50 \mathrm{~mL}$ de água e com $2 \mathrm{~h}$ de contato da água com a semente de quinoa.

Tabela 3 - Coeficientes correlação de Pearson, entre os tratamentos de condutividade elétrica e os testes de primeira contagem de germinação (PC), germinação $(\mathrm{G})$ e emergência de plântulas em substrato $(\mathrm{ESC})$ com sementes de quinoa $(C$. quinoa).

\begin{tabular}{|c|c|c|c|c|c|}
\hline \multicolumn{6}{|c|}{ Períodos de embebição (h) } \\
\hline Parâmetros & 1 & 2 & 3 & 4 & 5 \\
\hline \multicolumn{6}{|c|}{25 sementes / $25 \mathrm{~mL}$} \\
\hline $\mathrm{PC}$ & $-0,71^{*}$ & $-0,72^{*}$ & $-0,67 *$ & $-0,54^{\mathrm{ns}}$ & $-0,51^{\mathrm{ns}}$ \\
\hline G & $-0,69^{*}$ & $-0,69^{*}$ & $-0,64^{\mathrm{ns}}$ & $-0,52^{\mathrm{ns}}$ & $-0,50^{\mathrm{ns}}$ \\
\hline ESC & $-0,71^{*}$ & $-0,73^{*}$ & $-0,69 *$ & $-0,57^{\mathrm{ns}}$ & $-0,50^{\text {ns }}$ \\
\hline \multicolumn{6}{|c|}{25 sementes / $50 \mathrm{~mL}$} \\
\hline $\mathrm{PC}$ & $-0,70^{*}$ & $-0,70^{*}$ & $-0,51^{\mathrm{ns}}$ & $-0,63^{\mathrm{ns}}$ & $-0,56^{\mathrm{ns}}$ \\
\hline G & $-0,67^{*}$ & $-0,67^{*}$ & $-0,50^{\mathrm{ns}}$ & $-0,61^{\mathrm{ns}}$ & $-0,55^{\mathrm{ns}}$ \\
\hline ESC & $-0,87 * *$ & $-0,82 * *$ & $-0,70^{*}$ & $-0,75 * *$ & $-0,70 *$ \\
\hline \multicolumn{6}{|c|}{50 sementes / $25 \mathrm{~mL}$} \\
\hline $\mathrm{PC}$ & $-0,82 * *$ & $-0,80 * *$ & $-0,79 * *$ & $-0,79 *$ & $-0,79 * *$ \\
\hline G & $-0,83 * *$ & $-0,75^{* *}$ & $-0,72 *$ & $-0,73^{*}$ & $-0,73 *$ \\
\hline ESC & $-0,83 * *$ & $-0,79 * *$ & $-0,79 * *$ & $-0,81 * *$ & $-0,84 * *$ \\
\hline \multicolumn{6}{|c|}{50 sementes / $50 \mathrm{~mL}$} \\
\hline $\mathrm{PC}$ & $-0,42^{\text {ns }}$ & $-0,83 * *$ & $-0,75^{* *}$ & $-0,72^{*}$ & $-0,66^{*}$ \\
\hline G & $-0,3^{7 \mathrm{~ns}}$ & $-0,75^{* *}$ & $-0,71^{*}$ & $-0,69 *$ & $-0,64^{\mathrm{ns}}$ \\
\hline ESC & $-0,47^{\mathrm{ns}}$ & $-0,84 * *$ & $-0,84 * *$ & $-0,79 * *$ & $-0,72 *$ \\
\hline \multicolumn{6}{|c|}{75 sementes / $25 \mathrm{~mL}$} \\
\hline $\mathrm{PC}$ & $-0,51^{\mathrm{ns}}$ & $-0,57^{\mathrm{ns}}$ & $-0,52^{\mathrm{ns}}$ & $-0,54^{\mathrm{ns}}$ & $-0,53^{\mathrm{ns}}$ \\
\hline G & $-0,50^{\mathrm{ns}}$ & $-0,55^{\mathrm{ns}}$ & $-0,50^{\mathrm{ns}}$ & $-0,50^{\mathrm{ns}}$ & $-0,49^{\mathrm{ns}}$ \\
\hline ESC & $-0,63^{\mathrm{ns}}$ & $-0,74^{*}$ & $-0,69^{*}$ & $-0,71 *$ & $-0,68^{*}$ \\
\hline \multicolumn{6}{|c|}{75 sementes / $50 \mathrm{~mL}$} \\
\hline $\mathrm{PC}$ & $-0,66^{\mathrm{ns}}$ & $-0,70^{*}$ & $-0,74^{*}$ & $-0,68 *$ & $-0,69 *$ \\
\hline G & $-0,67 *$ & $-0,68^{*}$ & $-0,72 *$ & $-0,67 *$ & $-0,68^{*}$ \\
\hline ESC & $-0,89 * *$ & $-0,93 * *$ & $-0,87 * *$ & $-0,83^{* *}$ & $-0,84 * *$ \\
\hline
\end{tabular}

${ }^{\mathrm{ns}}$ não significativo, ${ }^{* *}$ significativo $(\mathrm{p}<0,01) \mathrm{e}$ *significativo $(\mathrm{p}<0,05)$ pelo teste t. Fonte: Autores.

Com base nos resultados (Tabela 3), pode-se afirmar que o teste de CE foi eficiente na separação dos lotes em níveis de vigor em sementes de quinoa, mediante o uso de 50 sementes em $50 \mathrm{~mL}$ de água deionizada em 2 h de embebição havendo uma relação entre o teste de emergência de plântulas.

A relevância dessa constatação pode ser verificada na afirmação de Marcos Filho (2015), de que reduzir período necessário para condução do teste é vantajoso para todo o setor envolvido com sementes, pois permite agilizar as tomadas de decisão referentes ao manejo dos lotes. Nesse sentido, o teste de CE cumpre com sua finalidade em detectar diferenças na qualidade 
entre lotes de quinoa, classificando-os em diferentes níveis de vigor, alto, médio e baixo de maneira proporcional à emergência das plântulas.

\section{Conclusão}

$\mathrm{O}$ teste de condutividade elétrica conduzido com 50 sementes embebidas em $50 \mathrm{~mL}$ de água deionizada, durante $2 \mathrm{~h}$, é eficiente em identificar diferenças de vigor entre os lotes de sementes de quinoa.

\section{Agradecimentos}

A CAPES (Coordenação de Aperfeiçoamento de Pessoal de Nível Superior) pelo incentivo e financiamento deste trabalho e, ao Programa de Pós-Graduação em Agronomia da Universidade Federal de Santa Maria.

\section{Referências}

Araujo, R. F., Zonta, J. B., Araujo, E. F., Herberle, E., \& Zonta, F. M. G. (2011). Teste de condutividade elétrica para sementes de feijão-mungo-verde. Revista Brasileira de Sementes, 33, (1), 123-130. 10.1590/S0101-31222011000100014

Bazile, D., Jacobsen, S. E., \& Verniau, A. (2016). The global expansion of quinoa: trends and limits. Frontiers in Plant Science, 7, 622. 10.3389/fpls.2016.00622

Borges, C. T. (2017). Desenvolvimento de metodologias para avaliação da qualidade fisiológica e conservação de sementes de quinoa (Chenopodium quinoa Willd.). 90 f. (Tese de Doutorado), Universidade Federal de Pelotas, Pelotas, Brasil.

Brasil. (2009). Regras para Análise de Sementes. Ministério da Agricultura, Pecuária e Abastecimento. Secretaria de Defesa Agropecuária, Brasília: MAPA.

Carvalho, N. M., \& Nakagawa, J. (2012). Sementes: ciência, tecnologia e produção. FUNEP.

Delouche, J. C; \& Baskin, C. C. (1973). Accelerated aging techniques for predicting the relative storability of seed lots. Seed Science and Technology, 1(3), 427-452.

Ferreira, D. F. (2014). Sisvar: A guide for its bootstrap procedures in multiple comparisons. Ciência e Agrotecnologia, 38, (2), 109-112. 10.1590/S141370542014000200001

Haesbaert, F. M., Lopes, S. J., Mertz, L. M., Dal'Col Lúcio, A., \& Huth, C. (2017). Tamanho de amostra de sementes de girassol. Bragantia, 76, (1), 54-61. $10.1590 / 1678-4499.389$

ISTA - International Seed Testing Association. (2014). Seed Vigour Testing. London: Internacional Seed Testing Association.

Lodo, M. A., Lopes, M. M., Soares, B. R. R., \& Vieira, R.D. (2013). Assessment of the physiological potential of spinach seeds (Tetragonia tetragonoides (Pall.) Kuntze). Journal of Seed Science, 35 (3), 284-291. 10.1590/S2317-15372013000300002

Marcos Filho, J. (2015). Fisiologia de sementes de plantas cultivadas. ABRATES.

Menezes, N. L., Garcia, D. C., Bahry, C. A., \& Mattioni, N. M. (2007). Teste de condutividade elétrica em aveia preta. Revista Brasileira de Sementes, 29(2), 138-142. 10.1590/S0101-31222007000200019

Murphy, K. M., Bazile, D., Kellogg, J., \& Rahmanian, M. (2016). Development of a Worldwide Consortium on Evolutionary Participatory Breeding in Quinoa. Frontiers in Plant Science, 9 (7), 608-628. 10.3389/fpls.2016.00608

Powell, A. A., \& Mavi, K. (2016). Application of the radicle emergence test to radish (Raphanus sativus) seed. London: Internacional Seed Testing Association.

R Core Team (2018). R: A language and environment for statistical computing. R Foundation for Statistical Computing.

Rosa, S. D. V., Renzo, G. V., Pinho, M. G. G. C., \& Veiga, R. D. (2000). Eficácia do teste de condutividade elétrica para uso em estudos de danos de secagem em sementes de milho. Revista Brasileira de Sementes, 22 (1), 54-63. 10.17801/0101-3122/rbs.v22n1p54-63

Spehar, C. R.; Rocha, J. E. S.; \& Santos, R. L. B. (2011). Desempenho agronômico e recomendações para cultivo de quinoa (BRS Syetetuba) no Cerrado. Pesquisa Agropecuária Tropical, 41 (1), 145-147. 10.5216/pat.v41i1.9395

Sponchiado, J. C., Souza, C. A., \& Coelho, C. M. M. (2014). Teste de condutividade elétrica para determinação do potencial fisiológico de sementes de aveia branca. Semina: Ciências Agrárias, 35(4), 2405-2414. 10.5433/1679-0359.2014v35n4Suplp2405

Torres, S. B., Dantas, A. H., Pereira, M. F. S., Benedito, C. P., \& Silva, F. H. S. (2012). Deterioração controlada em sementes de coentro. Revista Brasileira de Sementes, 34(2), 319-326. 10.1590/S0101-31222012000200018 
Research, Society and Development, v. 10, n. 5, e12510514682, 2021

(CC BY 4.0) | ISSN 2525-3409 | DOI: http://dx.doi.org/10.33448/rsd-v10i5.14682

Torres, S. B., Paiva, E. F., Almeida, J. P. N., Benedito, C. P., \& Carvalho, S. M. C. (2015). Teste de condutividade elétrica na avaliação da qualidade fisiológica de sementes de coentro. Revista Ciência Agronômica, 46 (3) 622-629. 10.5935/1806-6690.20150046

Torres, S. B.; \& Pereira, R. A. (2010.) Condutividade elétrica em sementes de rúcula. Revista Brasileira de Sementes, 32 (4), 58-70. 10.1590/S010131222010000400007

Vega-Galvez, A., Miranda, M., Vergara, J., Uribe, E. Puente, L., \& Martinez, E. A. (2010). Nutrition facts and functional potential of quinoa (Chenopodium quinoa Willd.), an ancient Andean grain: a review. Journal of the Science of Food and Agriculture, 90(15), 2541-2547. 10.1002 / jsfa.4158

Vilcacundo, R.; \& Hernández-Ledesma, B. (2017). Nutritional and Biological Value of Quinoa (Chenopodium quinoa Willd.). Current Opinion in Food Science, 14 (1), 1-6. 10.1016/j.cofs.2016.11.007 